\title{
Associations between $E R a / \beta$ gene polymorphisms and osteoporosis susceptibility and bone mineral density in postmenopausal women: a systematic review and meta-analysis
}

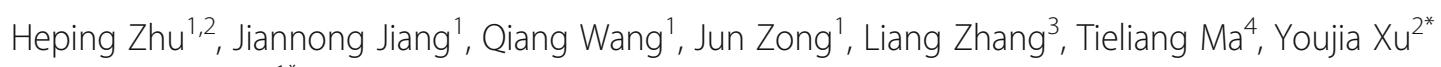
and Leiyan Zhang ${ }^{1 *}$

\begin{abstract}
Background: Many studies have reported associations between estrogen receptor (ER) gene polymorphisms and postmenopausal osteoporosis (PMOP) risk and bone mineral density (BMD), but the results are controversial. The aim of the present meta-analysis is to verify the association between ERa and ER $\beta$ gene polymorphisms and osteoporosis susceptibility and BMD in postmenopausal women.

Methods: PubMed, EMBASE, Web of Science, the Cochrane Library and China WeiPu Library were searched. OR and WMD with $95 \% \mathrm{Cl}$ were calculated to assess the association.

Results: Overall, no significant association was observed between ERa Xbal, ERa Pvull and PMOP susceptibility in either overall, Caucasian or Asian populations. ERa G2014A was significantly associated with a decreased risk of PMOP in Caucasian populations. There was a significant association between ER Rsal and PMOP risk in both overall and Asian populations. Caucasian PMOP women with ERa Xbal XX and Xx genotypes had a higher LS Z value than women with XX genotype. ERa Xbal XX genotype was associated with increased FN BMD in overall and Caucasian populations, an increased FN Z value in Asians, and a decreased FN Z value in Caucasians. There was also a significant association between ERa Xbal Xx genotype and an increased FN Z value in either Asians or Caucasians. ERa Pvull PP genotype was associated with a low LS Z value in Caucasians and a low FN BMD and $Z$ value in Asians. Pp genotype in PMOP women was significantly correlated with low LS BMD in overall populations, a low FN $Z$ value in either overall, Caucasian or Asian populations.
\end{abstract}

Conclusion: Each ERa and ER $\beta$ gene polymorphism might have different impact on PMOP risk and BMD in various ethnicities.

Keywords: Estrogen receptor, Postmenopausal osteoporosis, Gene polymorphism, Meta-analysis

\footnotetext{
*Correspondence: xuyoujia@suda.edu.cn; 2004zhp-pp@sohu.com

${ }^{2}$ Department of Orthopedics, The Second Affiliated Hospital of Soochow

University, Suzhou 215004, China

'Department of Orthopedics, The Affiliated Yixing Hospital of Jiangsu

University, Yixing 214200, China

Full list of author information is available at the end of the article
} 


\section{Background}

Postmenopausal osteoporosis (PMOP) is a common metabolic bone disorder characterized by low bone mineral density (BMD) and increased fracture risks [1-3]. It is estimated that osteoporosis affects approximately 10 million American adults, with another 34 million being at high risk due to low bone mass [4].

The pathophysiology of PMOP is considered as a disorder or negative imbalance of bone metabolism and remodeling, with bone resorption outpacing bone formation [3], suggesting that vitamin $\mathrm{D}$ and parathyroid hormone $(\mathrm{PTH})$ and other factors related to bone resorption and formation may play a key role in the underlying mechanism and pathophysiology of PMOP [5-8]. Furthermore, genetic factors including genes and gene polymorphisms may also play an important role in the development of PMOP [9].

Estrogen is another important hormone that plays an important role in the pathogenesis of PMOP, knowing that reduced ovarian production of estrogen after menopause is a cause for the initial phase of rapid bone loss and osteoporosis in women [3]. Estrogen is known as an important regulator of bone metabolism, and estrogen deficiency is believed to be the cause of BMD loss, increased mechanical loadinginduced bone remodeling, and the development of PMOP [10]. Knowing that the action of estrogen is predominantly mediated by estrogen receptor (ER), including $E R \alpha$ and $E R \beta$ by binding to different ligands to mediate various biological effects [3, 10], more attention has been paid to the relationship between ERs and PMOP risk and BMD in postmenopausal women [11-38]. However, the results of studies currently available about this issue are controversial.

Previous meta-analyses have been performed to assess the pooled effects of ER gene polymorphisms on BMD and fracture risk [39-41]. WANG et al. [39] showed that the ER $\alpha$ XbaI (rs9340799) polymorphism was associated with BMD at diverse skeletal sites, and ER $\alpha$ PvuII (rs2234693) PP genotype played a role in protecting the lumbar spine but on the other hand might be a risk factor for the femoral neck fracture. However, to the best of our knowledge, no meta-analysis has been performed to explore the relationships between ER gene [ER $\alpha \mathrm{XbaI}$ (rs9340799), ER $\alpha$ PvuII (rs2234693) and ER $\alpha$ G2014A (rs2228480)] and ER $\beta$ gene [ER $\beta$ AluI (rs4986938) and ER $\beta$ RsaI (rs1256049)] polymorphisms and PMOP susceptibility and BMD of the lumbar spine and femoral neck in postmenopausal women. To address these issues, we performed a meta-analysis of all currently available studies relating ER gene [ER $\alpha$ XbaI (rs9340799), ER $\alpha$ PvuII (rs2234693) and ER $\alpha$ G2014A (rs2228480)] and ER $\beta$ gene $[\mathrm{ER} \beta$ AluI (rs4986938) and ER $\beta$ RsaI (rs1256049)] polymorphisms with PMOP risk and BMD.

\section{Methods}

\section{Data sources and searches}

We searched PubMed, EMBASE, Web of Science, the Cochrane Library and China WeiPu Library to identify case-control studies that investigated the associations between ER $\alpha$ gene polymorphisms [ER $\alpha$ XbaI (rs9340799), ER $\alpha$ PvuII (rs2234693) and ER $\alpha$ G2014A (rs2228480)] ER $\beta$ gene polymorphisms [ER $\beta$ AluI (rs4986938) and ER $\beta$ RsaI (rs1256049)] and osteoporosis susceptibility and $\mathrm{BMD}$ in postmenopausal women by using the following search terms ('PMOP' OR 'Postmenopausal osteoporosis' OR 'Postmenopausal') AND ('Estrogen Receptor' OR 'ER') AND ('polymorphism' OR 'single nucleotide polymorphism' OR 'SNP' OR 'variation'). To analyze the pooled effects of ER gene polymorphisms on BMD, the following search terms were used: ('PMOP' OR 'Postmenopausal osteoporosis' OR 'Postmenopausal') AND ('Estrogen Receptor' OR 'ER') AND ('polymorphism' OR 'single nucleotide polymorphism' OR 'SNP' OR 'variation') AND ('BMD' OR 'bone mineral density'). Then, one-by-one screening was performed by two authors according to the inclusion and exclusion criteria. No language restrictions were applied. Secondary searches of eligible studies were conducted by searching the reference lists of the selected studies, reviews or comments.

\section{Inclusion and exclusion criteria}

The inclusion criteria of our meta-analysis are as follows: (1) case-control studies; (2) studies on BMD and fracture risks in postmenopausal women with PMOP due to estrogen deficiency using postmenopausal women without PMOP or healthy volunteers as control; (3) studies reporting alleles and genotypes of at least one of the ER gene polymorphisms in women with or without PMOP: ER $\alpha$ XbaI (rs9340799), ER $\alpha$ PvuII (rs2234693), ER $\alpha$ G2014A (rs2228480), ER $\beta$ AluI (rs4986938) and ER $\beta$ RsaI (rs1256049); (3) studies reporting the sample size, mean and standard deviation (SD) of BMD $\left(\mathrm{g} / \mathrm{cm}^{2}\right)$ or $\mathrm{BMD} \mathrm{Z}$ value in PMOP women with at least one of the ER genotypes; and (4) studies with sufficient data. The exclusion criteria were: (1) reviews or case reports without controls, and (2) studies with no availability of current data; and (3) duplicated reports.

\section{Data extraction}

Data from the eligible studies were extracted according to the inclusion and exclusion criteria by two authors, and a consensus was reached by discussion. In the study of associations between ER gene polymorphisms and PMOP risk, the following data were collected: author list, year of publication, ethnicity, sample size, alleles, genotype of each gene polymorphism and HardyWeinberg equilibrium (HWE). The following data were collected for analysis of differences in BMD in PMOP 
women with various ER genotypes: author list, year of publication, ethnicity, the number of cases and mean and $\mathrm{SD}$ of BMD $\left(\mathrm{g} / \mathrm{cm}^{2}\right)$ and BMD $\mathrm{Z}$ value.

\section{Data synthesis and statistical analysis}

We calculated odds ratios (OR) and 95\% confidence interval $(\mathrm{CI})$ to evaluate the association between ER gene polymorphisms and PMOP risk (osteoporosis occurred in postmenopausal women due to estrogen deficiency as represented by low BMD and increased fracture risks). The strength of association between ER gene polymorphisms and PMOP susceptibility was evaluated by OR and $95 \% \mathrm{CI}$ under the allele contrast model, heterozygote model, homozygote model, dominant model and recessive model. HWE was calculated in the control population to evaluate the quality of the data by using chisquare test. Regarding the associations between BMD and ER gene polymorphisms, we compared BMD (g/ $\mathrm{cm}^{2}$ ) and BMD $\mathrm{Z}$ value in PMOP women under the heterozygote and homozygote model respectively using the weight mean difference (WMD) and 95\% CI. Heterogeneity of the included studies was examined by a chisquared-based $\mathrm{Q}$ statistical test and quantified by I2 metric value. If I2 value was $>50 \%$ or $P<0.10$, ORs and WMD were pooled by the random effect model; otherwise, the fixed effect model was used. Power analysis was performed using the Power and Precision V4 software (Biostat Inc., Englewood, USA). Sensitivity analysis was performed to assess the impact of each study on the combined effect of the present meta-analysis. Besides, subgroup analysis was also performed according to the ethnicity of the study populations. Stata 12.0 software (StataCorp, College Station, TX, USA) was used and a $P$ $<0.05$ was considered as statistically significant.

\section{Results}

Study selection and characteristics

A total of 28 studies [11-38] were finally recruited in our meta-analysis. The study selection and inclusion process is shown in Fig. 1. Fourteen studies [11-24]

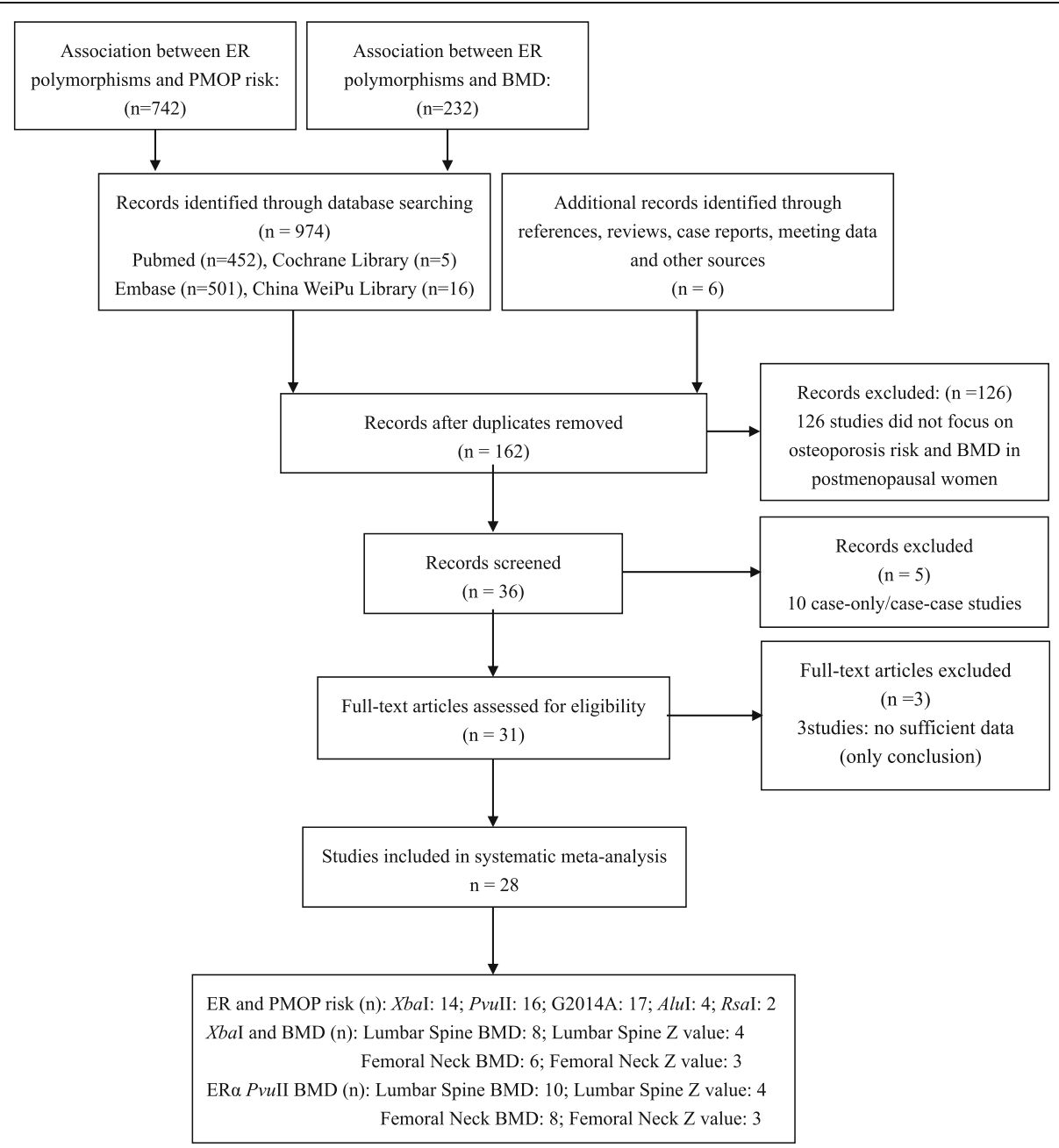

Fig. 1 Flow chart showing the process of selection 
reported the association between ER $\alpha \mathrm{Xba \textrm {I }}$ and PMOP risk, and the number of the included studies that reported the alleles and genotypes of ER $\alpha P v u I I, E R \alpha$ G2014A, ER $\beta$ AluI and ER $\beta$ RsaI was 16 [11-25, 32], 4 [26-29], 4 [17, 30-32] and 2 [30, 31], respectively. Ivanova et al. [20], Albagha et al. [33], Aerssens et al. [24], Kurt et al. [34], Ge et al. [36] and Pérez et al. [19] reported both the lumbar spine and femoral neck BMD $\left(\mathrm{g} / \mathrm{cm}^{2}\right)$. Jeedigunta et al. [15] and Kurabayashi et al. [35] were also recruited in the assessment of the lumbar spine BMD $\left(\mathrm{g} / \mathrm{cm}^{2}\right)$ in ER $\alpha b a \mathrm{I}$ genotypes. Ivanova et al. [20], Albagha et al. [33] and An et al. [38] reported both the lumbar spine and femoral neck $\mathrm{Z}$ values. Shang et al. [11] also studied the lumbar spine $Z$ value in PMOP with ER $\alpha$ XbaI genotypes. Ten studies [15, 19, $20,23,24,33-37]$ and 8 studies $[19,20,23,24,33,34$, $36,37]$ were recruited in the pooled analysis of differences in lumbar spine and femoral neck BMD $\left(\mathrm{g} / \mathrm{cm}^{2}\right)$ in PMOP women carrying ER $\alpha$ PvuII, respectively. With regard to differences in lumbar spine and femoral neck $\mathrm{Z}$ value in PMOP women with ER $\alpha$ PvuII, 4 studies [11, $20,33,38]$ and 3 studies [20,33, 38] were included in our meta-analysis, respectively. In addition, all these studies complied with HWE. The characteristics of the included studies are shown in Tables 1, 2 and 3.

\section{Power analysis}

Before initiation of the meta-analysis, a power analysis was conducted by using the Power and Precision V4 software to verify whether the included studies could offer adequate power $(>80 \%)$. The result showed that the statistical power in our study was sufficient to detect the associations between ER gene polymorphisms and PMOP risk.

\section{Associations between ER gene polymorphisms and PMOP risk}

Overall, we did not find any significant association be-

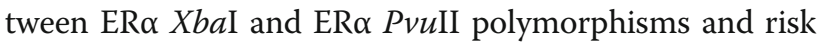
of PMOP in either overall, Caucasian or Asian populations (all $P>0.05$ ) (Table 4). ER $\alpha$ G2014A polymorphism played a protcetive role in developing PMOP in Caucasian populations, while no significant association was observed in overall and Asian populations (both $P>$ 0.05). All the data are shown in Table 4 and Fig. 2.

With regard to ER $\beta$ polymorphism, ER $\beta$ AluI was significantly associated with the risk of developing PMOP in Asian postmenopausal women under the recessive model; however, we did not observe any significant association between ER $\beta$ AluI and PMOP risk in overall and Caucasian populations (both $P>0.05$ ) (Table 4 and Fig. 3). Furthermore, we also found that there was a remarkable association between ER $\beta$ RsaI polymorphism and decreased PMOP risk in overall and Asian populations (Table 4).

\section{Associations between ER gene polymorphisms and BMD in PMOP women \\ $E R a X b a l$ and lumbar spine bone mineral density (BMD g/ $\mathrm{cm}^{2}$ and $B M D Z$ value)}

In our meta-analysis, no significant difference in lumbar spine BMD $\left(\mathrm{g} / \mathrm{cm}^{2}\right)$ was observed between PMOP women with ER $\alpha$ XbaI XX, ER $\alpha$ XbaI Xx and ER $\alpha b a \mathrm{I}$ $\mathrm{xx}$ genotype in either overall, Caucasian or Asian populations (all $P>0.05$ ) (Table 5). The lumbar spine BMD Z value in Caucasian PMOP women carrying ER $\alpha \mathrm{XbaI}$ $\mathrm{XX}$ genotype was greater than that in those carrying $\mathrm{Xx}$ genotype, while no significant difference was observed in overall and Asian populations (both $P>0.05$ ). ER $\alpha$ $\mathrm{XbaI} \mathrm{Xx}$ genotype was found to be significantly associated with high lumbar spine BMD $\mathrm{Z}$ value in either overall or Caucasian populations but not in Asian populations.

\section{ERa Xbal and femoral neck bone mineral density (BMD g/ $\mathrm{cm}^{2}$ and $B M D Z$ value)}

Our pooled analyses indicated that the ER $\alpha \mathrm{XbaI} X X$ genotype was significantly associated with increased femoral neck BMD in overall and Caucasian popula-

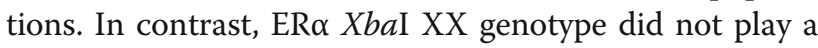
key role in femoral neck BMD in Asian populations (Table 5 and Fig. 4). Interestingly, compared with PMOP women with $\mathrm{xx}$ genotype, $\mathrm{XX}$ genotype was significantly associated with decreased femoral neck $Z$ value in Caucasians, and increased femoral neck $\mathrm{Z}$ value in Asians (Table 5). However, no significant association was observed between XX genotype and the femoral neck $\mathrm{Z}$ value in overall populations. In addition, Caucasians and Asians carrying the ER $\alpha \mathrm{X} b a \mathrm{I} \mathrm{Xx}$ genotype were at risk of a high femoral neck $Z$ value, while no significant association was found in overall populations. We did not observe remarkable relationships between ER $\alpha$ XbaI Xx genotype and femoral neck BMD in either overall, Caucasian or Asian populations (all $P>0.05$ ). All data are shown in Table 5.

\section{ERa Pvull and lumbar spine bone mineral density (BMD g/ $\mathrm{cm}^{2}$ and $B M D Z$ value)}

With regard to ER $\alpha$ PvuII, the difference in the lumbar spine $\mathrm{Z}$ value between the PP and pp. genotypes was $0.07(95 \% \mathrm{CI}=-0.03$ to $-0.01, P=0.031)$ in Caucasian PMOP women; however, no significant difference was observed in overall and Asian populations. For the Pp versus pp. genotype, the difference in lumbar spine BMD was $0.01(95 \% \mathrm{CI}=-0.02$ to $-0.00, P=0.036)$ in overall populations, and the difference in the lumbar spine $\mathrm{Z}$ value was $-0.16(95 \% \mathrm{CI}=-0.20$ to $-0.12, P<0.001)$ in Caucasian 
Table 1 General characteristics of studies assciated with postmenopausal osteoporosis risk

\begin{tabular}{|c|c|c|c|c|c|c|c|c|c|c|c|c|c|c|c|}
\hline \multirow[t]{3}{*}{ Author } & \multirow[t]{3}{*}{ Year } & \multirow[t]{3}{*}{ Ethnicity } & \multicolumn{2}{|c|}{ Sample Size } & \multicolumn{10}{|c|}{ ERa Xbal } & \multirow[t]{3}{*}{ HWE } \\
\hline & & & & & \multicolumn{5}{|l|}{ Case } & \multicolumn{5}{|c|}{ Control } & \\
\hline & & & Case & Control & $x$ & $x$ & $X X$ & $X x$ & $x x$ & $x$ & $x$ & $X X$ & $X x$ & $x x$ & \\
\hline Shang et al. & 2016 & Asian & 198 & 276 & 338 & 58 & 146 & 46 & 6 & 109 & 443 & 10 & 89 & 177 & 0.77 \\
\hline Wang et al. & 2015 & Asian & 72 & 72 & 125 & 19 & 55 & 15 & 2 & 132 & 12 & 62 & 8 & 2 & 0.21 \\
\hline Li et al. & 2014 & Asian & 440 & 791 & 254 & 626 & 31 & 192 & 217 & 404 & 1178 & 48 & 308 & 435 & 0.50 \\
\hline Erdogan et al. & 2011 & Caucasian & 50 & 30 & 41 & 59 & 7 & 27 & 16 & 28 & 32 & 6 & 16 & 8 & 0.70 \\
\hline Jeedigunta et al. & 2010 & Asian & 247 & 254 & 253 & 241 & 60 & 133 & 54 & 306 & 202 & 81 & 144 & 29 & 0.32 \\
\hline Tanriover et al. & 2010 & Caucasian & 50 & 50 & 48 & 52 & 5 & 38 & 7 & 54 & 46 & 12 & 30 & 8 & 0.14 \\
\hline Harsløf et al. & 2010 & Caucasian & 228 & 225 & 134 & 322 & 19 & 96 & 113 & 164 & 286 & 30 & 104 & 91 & 0.97 \\
\hline Musumeci et al. & 2009 & Caucasian & 100 & 200 & 130 & 70 & 35 & 60 & 5 & 155 & 245 & 13 & 129 & 58 & 0.26 \\
\hline Pérez et al. & 2008 & Caucasian & 64 & 68 & 48 & 80 & 9 & 30 & 25 & 46 & 90 & 5 & 36 & 27 & 0.13 \\
\hline Ivanova et al. & 2007 & Caucasian & 220 & 180 & 256 & 184 & 73 & 110 & 37 & 163 & 197 & 25 & 113 & 42 & 0.58 \\
\hline Huang et al. & 2006 & Asian & 66 & 116 & 19 & 113 & 2 & 15 & 49 & 46 & 186 & 4 & 38 & 74 & 0.74 \\
\hline Nam et al. & 2005 & Asian & 6 & 168 & 0 & 12 & 0 & 0 & 6 & 63 & 273 & 6 & 51 & 111 & 0.96 \\
\hline Qin et al. & 2004 & Asian & 244 & 273 & 120 & 368 & 11 & 98 & 135 & 137 & 409 & 13 & 111 & 149 & 0.18 \\
\hline Aerssens et al. & 2000 & Caucasian & 135 & 239 & 92 & 178 & 14 & 64 & 57 & 175 & 303 & 32 & 111 & 96 & 0.99 \\
\hline \multirow[t]{3}{*}{ Author } & Year & Ethnicity & \multirow{2}{*}{\multicolumn{2}{|c|}{ Sample Size }} & \multicolumn{5}{|c|}{ ERa Pvull } & & & & & & HWE \\
\hline & & & & & Case & & & & & \multicolumn{5}{|c|}{ Control } & \\
\hline & & & Case & Control & $P$ & $\mathrm{p}$ & PP & $\mathrm{Pp}$ & $\mathrm{pp}$ & $P$ & $\mathrm{p}$ & PP & $\mathrm{Pp}$ & $\mathrm{pp}$ & \\
\hline Shang et al. & 2016 & Asian & 198 & 276 & 156 & 240 & 28 & 100 & 70 & 386 & 166 & 138 & 110 & 28 & 0.38 \\
\hline Wang et al. & 2015 & Asian & 60 & 60 & 30 & 90 & 3 & 24 & 33 & 32 & 88 & 3 & 26 & 31 & 0.40 \\
\hline Li et al. & 2014 & Asian & 440 & 791 & 368 & 512 & 65 & 238 & 137 & 498 & 1084 & 69 & 360 & 362 & 0.12 \\
\hline Sonoda et al. & 2012 & Asian & 114 & 171 & 118 & 110 & 24 & 70 & 20 & 137 & 205 & 31 & 75 & 65 & 0.26 \\
\hline Erdogan et al. & 2011 & Caucasian & 50 & 30 & 42 & 58 & 8 & 26 & 16 & 38 & 22 & 10 & 18 & 2 & 0.11 \\
\hline Jeedigunta et al. & 2010 & Asian & 247 & 254 & 181 & 313 & 50 & 81 & 116 & 232 & 276 & 60 & 112 & 82 & 0.08 \\
\hline Tanriover et al. & 2010 & Caucasian & 50 & 50 & 39 & 61 & 7 & 25 & 18 & 48 & 52 & 14 & 20 & 16 & 0.79 \\
\hline Harsløf et al. & 2010 & Caucasian & 228 & 224 & 198 & 258 & 46 & 106 & 76 & 233 & 215 & 63 & 107 & 54 & 0.52 \\
\hline Musumeci et al. & 2009 & Caucasian & 100 & 200 & 120 & 80 & 30 & 60 & 10 & 186 & 214 & 31 & 124 & 45 & 0.53 \\
\hline Pérez et al. & 2008 & Caucasian & 64 & 68 & 56 & 72 & 11 & 34 & 19 & 58 & 78 & 12 & 34 & 22 & 0.86 \\
\hline Ivanova et al. & 2007 & Caucasian & 220 & 180 & 226 & 214 & 58 & 110 & 52 & 148 & 212 & 21 & 106 & 53 & 0.37 \\
\hline Morón et al. & 2006 & Caucasian & 87 & 175 & 79 & 95 & 17 & 45 & 25 & 171 & 179 & 45 & 81 & 49 & 0.33 \\
\hline Huang et al. & 2006 & Asian & 66 & 116 & 79 & 53 & 23 & 33 & 10 & 68 & 164 & 11 & 46 & 59 & 0.64 \\
\hline Nam et al. & 2005 & Asian & 6 & 168 & 2 & 10 & 1 & 0 & 5 & 130 & 206 & 25 & 80 & 63 & 0.96 \\
\hline Qin et al. & 2004 & Asian & 244 & 273 & 193 & 295 & 40 & 113 & 91 & 223 & 323 & 43 & 137 & 93 & 0.52 \\
\hline Aerssens et al. & 2000 & Caucasian & 135 & 239 & 120 & 150 & 27 & 66 & 42 & 219 & 259 & 47 & 125 & 67 & 0.41 \\
\hline \multirow[t]{3}{*}{ Author } & Year & Ethnicity & \multirow{2}{*}{\multicolumn{2}{|c|}{ Sample Size }} & \multicolumn{5}{|c|}{ ERa G2014A } & & & & & & HWE \\
\hline & & & & & Case & & & & & \multicolumn{5}{|c|}{ Control } & \\
\hline & & & Case & Control & A & G & AA & GA & GG & A & G & AA & GA & GG & \\
\hline Wajanavisit et al. & 2015 & Asian & 99 & 113 & 94 & 104 & 33 & 28 & 38 & 179 & 47 & 72 & 35 & 6 & 0.53 \\
\hline Gómez et al. & 2007 & Caucasian & 70 & 500 & 30 & 110 & 2 & 26 & 42 & 303 & 697 & 40 & 223 & 237 & 0.21 \\
\hline Ongphiphadhanakul et al. & 2003 & Asian & 33 & 325 & 23 & 43 & 5 & 13 & 15 & 129 & 521 & 13 & 103 & 209 & 0.94 \\
\hline Ongphiphadhanakul et al. & 2001 & Asian & 106 & 122 & 56 & 156 & 8 & 40 & 58 & 37 & 207 & 2 & 33 & 87 & 0.57 \\
\hline
\end{tabular}


Table 1 General characteristics of studies assciated with postmenopausal osteoporosis risk (Continued)

\begin{tabular}{|c|c|c|c|c|c|c|c|c|c|c|c|c|c|c|c|}
\hline \multirow[t]{3}{*}{ Author } & \multirow[t]{3}{*}{ Year } & \multirow[t]{3}{*}{ Ethnicity } & \multirow{2}{*}{\multicolumn{2}{|c|}{ Sample Size }} & \multicolumn{10}{|c|}{ ERß Alul } & HWE \\
\hline & & & & & \multicolumn{5}{|c|}{ Case } & \multicolumn{5}{|c|}{ Control } & \\
\hline & & & Case & Control & A & G & $A A$ & GA & GG & A & G & $A A$ & GA & GG & \\
\hline Shoukry et al. & 2015 & Caucasian & 200 & 180 & 223 & 177 & 75 & 73 & 52 & 125 & 235 & 30 & 65 & 85 & 0.46 \\
\hline Huang et al. & 2015 & Asian & 413 & 890 & 678 & 148 & 285 & 108 & 20 & 1384 & 396 & 541 & 302 & 47 & 0.57 \\
\hline Harsløf et al. & 2010 & Caucasian & 228 & 224 & 154 & 302 & 26 & 102 & 100 & 186 & 262 & 35 & 116 & 73 & 0.32 \\
\hline Morón et al. & 2006 & Caucasian & 88 & 177 & 76 & 100 & 11 & 54 & 23 & 146 & 208 & 34 & 78 & 65 & 0.23 \\
\hline \multirow[t]{3}{*}{ Author } & Year & Ethnicity & \multirow{2}{*}{\multicolumn{2}{|c|}{ Sample Size }} & \multicolumn{5}{|c|}{ ERß Rsal } & & & & & & \\
\hline & & & & & Case & & & & & \multicolumn{5}{|c|}{ Control } & \\
\hline & & & Case & Control & $A$ & G & AA & GA & GG & A & G & AA & GA & GG & \\
\hline Shoukry et al. & 2015 & Caucasian & 200 & 180 & 52 & 348 & 2 & 48 & 150 & 37 & 323 & 1 & 35 & 144 & 0.47 \\
\hline Huang et al. & 2015 & Asian & 413 & 777 & 329 & 497 & 63 & 203 & 147 & 759 & 795 & 169 & 421 & 187 & 0.28 \\
\hline
\end{tabular}

populations; however, we did not find any significant difference in lumbar spine BMD in either Caucasians or Asians, and in the lumbar spine $\mathrm{Z}$ value in overall and Asian populations (Table 5 and Fig. 5). In addition, no significant difference in lumbar spine BMD was observed between PP and pp. genotypes $(P>0.05)$ (Table 5).

\section{ERa Pvull and femoral neck bone mineral density (BMD g/ $\mathrm{Cm}^{2}$ and $B M D Z$ value)}

We further found that the ER $\alpha$ PvuII PP genotype was associated with decreased femoral neck BMD and Z value compared with the pp. genotype in Asians, while no significant difference in femoral neck BMD and $\mathrm{Z}$ value was observed in either overall and Caucasian populations (both $P>0.05$ ) (Table 5). Furthrmore, PMOP women carrying the $\mathrm{Pp}$ genotype were at risk of a low femoral neck $\mathrm{Z}$ value, which was found in overall, Caucasian and Asian populations. Our study showed that there was no significant difference in femoral neck BMD between PMOP women with the Pp genotype and those with the pp. genotype $(P>0.05)$. All the data are shown in Table 5.

\section{Sensitivity analysis and publication bias}

We performed a leave-one-out analysis to estimate the sensitivity of our study and found that omission of any single study did not affect the overall statistical significance, indicating that the results of our metaanalysis are stable. Therefore, we could conclude that our meta-analysis data are relatively stable and credible. To estimate the publication bias of our metaanalysis, the Begg's and Egger's test was performed (Table 4), indicating that there was minimal evidence of publication bias. The shape of funnel plot was symmetrical, which also showed no publication bias in our study (Fig. 6).

\section{Discussion}

Associations between ERa gene polymorphisms and PMOP risk

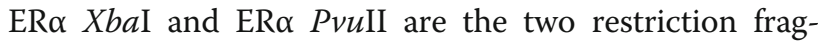
ment length polymorphisms of ER $\alpha$ gene located in Intron 1 [14]. Many studies [11-25, 32] have been performed to explore the relationships between ER $\alpha$ $X b a \mathrm{I}, \mathrm{ER} \alpha$ PvuII and PMOP risk; however, these studies have yielded inconsistent data [11-25, 32]. Overall, we did not observe any significant association between ER $\alpha$

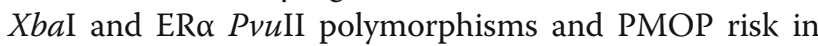
either overall, Caucasian or Asian populations. In our opinion, the inadequate sample size, different ethnicities, various genotyping techniques, the presence of admixture in the population, gene-environment interactions, differences in age and measurement errors of different investigators might be important factors contributing to these controversial results. ER $\alpha \quad X b a \mathrm{I}$ and ER $\alpha$ PvuII have proven to play key roles in attainment and maintenance of peek bone mass during young adulthood, and it might be difficult to document their effects in a population of postmenopausal women [24]. In addition, PvuII and $\mathrm{XbaI}$ polymorphisms are located in a non-functional area of the ER gene [20], which might also contribute to our polled results. With regard to ER $\alpha \mathrm{G} 2014 \mathrm{~A}$, it is located on the exon region of chromosome 6p25.1, and may contribute via the epigenetic level for the efficiency of translation or receptor protein expression [26]. Our results showed that a significant association between $\mathrm{ER} \alpha \mathrm{G} 2014 \mathrm{~A}$ and PMOP risk was observed only in Caucasian populations but not in overall and Asian populations.

\section{Associations between ER $\beta$ gene polymorphisms and PMOP risk}

ER $\beta$ has been found to be more abundant than ER $\alpha$ in trabecular bone, and more potent than ER $\alpha$ in 


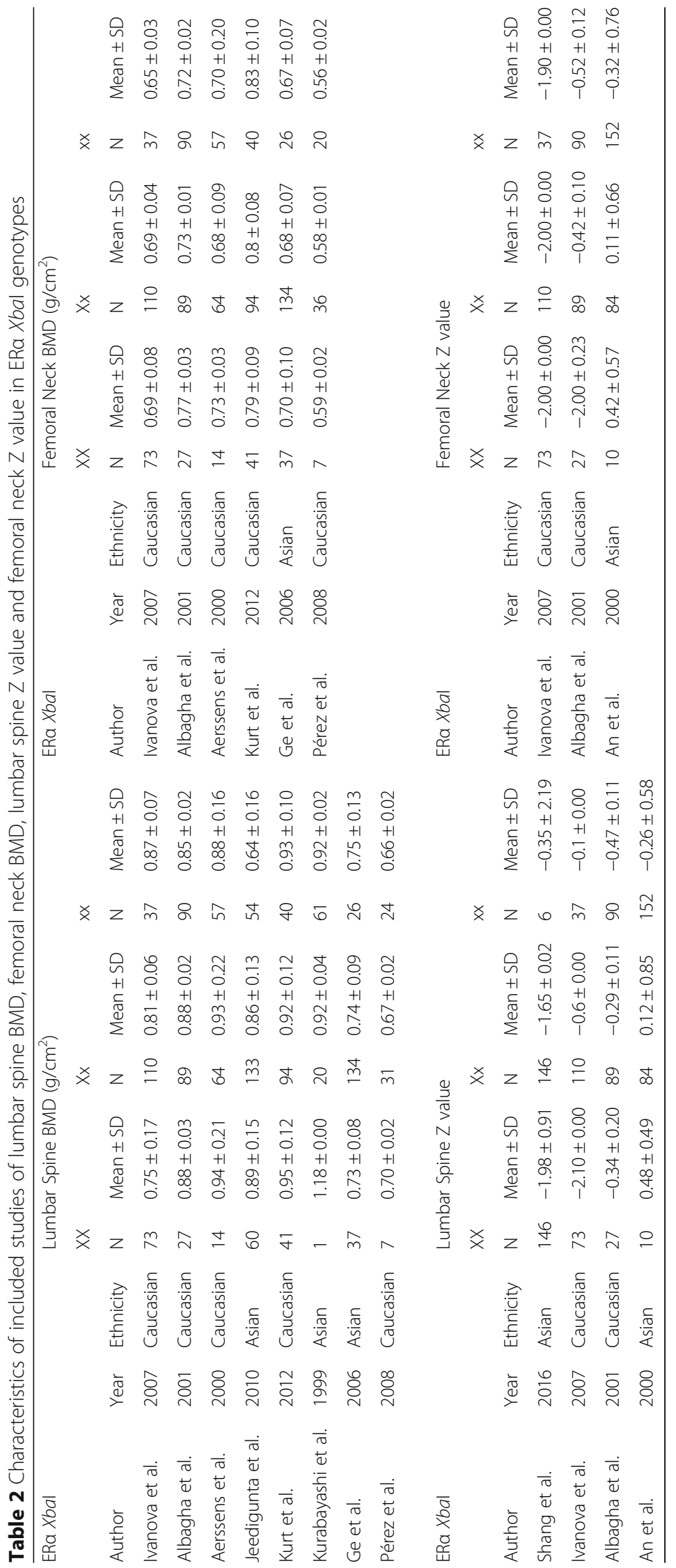




\section{要

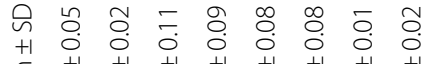

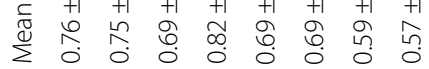

은

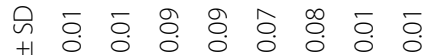

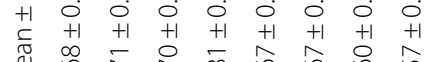

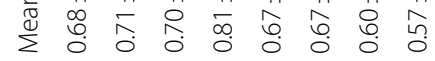

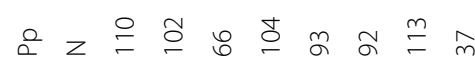

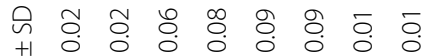

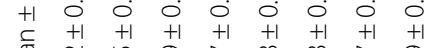

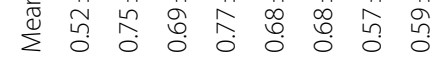

a $z \stackrel{\infty}{n} \hat{m} \forall \stackrel{\infty}{m}$ ㅇ $a$

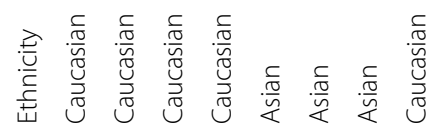

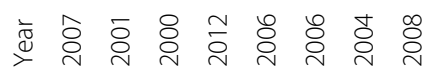

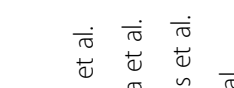

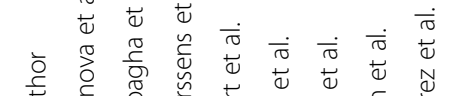

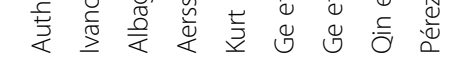

की

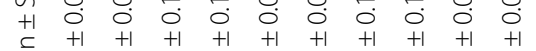

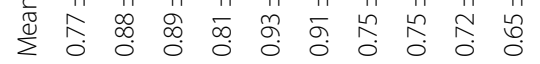

$\AA z$ กิ่์

ใิ 응 ส

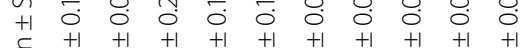

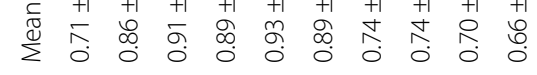

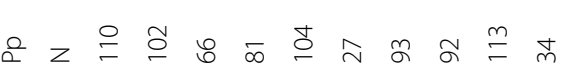

in o.

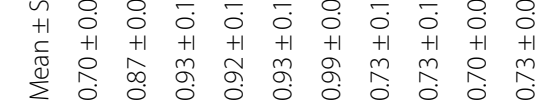

a $z \stackrel{\infty}{n} \hat{\sim}$ i $j a \stackrel{\infty}{m} \underset{m}{\infty}=$

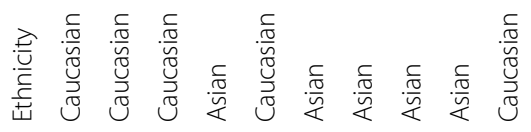

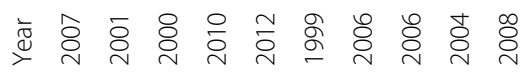

in $\frac{8}{0} \stackrel{0}{\circ} \frac{0}{\circ}$

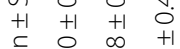

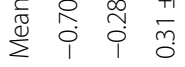

$\stackrel{\circ}{2} \sim \hat{\sigma} \stackrel{\infty}{\simeq}$

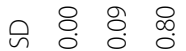

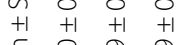

焉

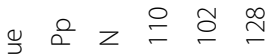

$\frac{\sqrt{2}}{3}$

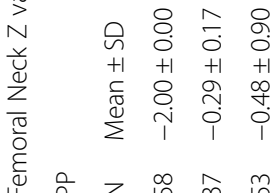

a $z$ in $\bar{m} n$

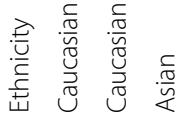

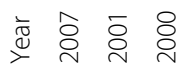

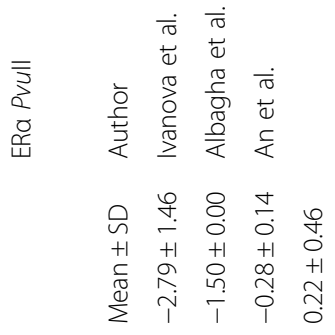

$\therefore z R$ ก

จ

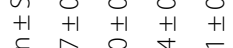

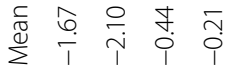

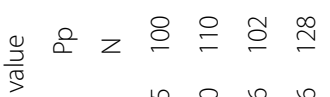

ก

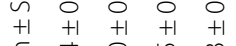

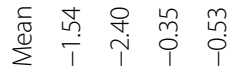

a $z \stackrel{\infty}{\sim} \stackrel{\infty}{i n} n$

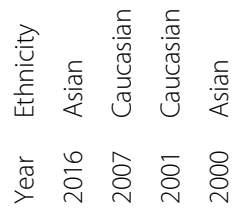

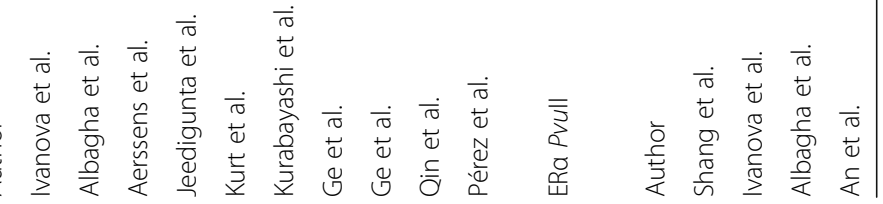


Table 4 Results of genetic models for ERa Xbal, ERa Pvull, ERa G2014A, ERß Alul and ER $\beta$ Rsal polymorphisms and osteoporosis susceptibility in postmenopausal women

\begin{tabular}{|c|c|c|c|c|c|c|c|c|c|}
\hline \multirow[t]{2}{*}{ Comparison } & \multirow[t]{2}{*}{$\mathrm{N}$} & \multicolumn{3}{|c|}{ Test of association } & \multirow[t]{2}{*}{ Model } & \multicolumn{2}{|c|}{ Test of heterogeneity } & \multirow{2}{*}{$\begin{array}{l}\text { Begg's test } \\
P \text { value }\end{array}$} & \multirow{2}{*}{$\begin{array}{l}\text { Egger's test } \\
P \text { value }\end{array}$} \\
\hline & & $\overline{\mathrm{OR}}$ & $95 \% \mathrm{Cl}$ & $P$ value & & $\overline{P \text { value }}$ & $\mathrm{I}^{2}(\%)$ & & \\
\hline \multicolumn{10}{|l|}{ ERa Xbal } \\
\hline Overall & 14 & & & & & & & & \\
\hline$X$ vs. $X$ & & 1.21 & $0.73-2.00$ & 0.455 & $\mathrm{R}$ & $<0.001$ & 96.4 & 0.584 & 0.955 \\
\hline$X X$ vs. XX & & 1.84 & $0.71-4.75$ & 0.206 & $\mathrm{R}$ & $<0.001$ & 93.7 & 0.443 & 0.465 \\
\hline XX vs. Xx & & 1.19 & $0.83-1.70$ & 0.357 & $\mathrm{R}$ & $<0.001$ & 80.1 & 0.511 & 0.610 \\
\hline$X x / X X$ vs. $x X$ & & 1.34 & $0.82-2.18$ & 0.240 & $\mathrm{R}$ & $<0.001$ & 90.4 & 0.661 & 0.545 \\
\hline$X X$ vs. $X x / x X$ & & 1.50 & $0.70-3.24$ & 0.296 & $\mathrm{R}$ & $<0.001$ & 93.4 & 0.443 & 0.875 \\
\hline Caucasian & 7 & & & & & & & & \\
\hline X vs. X & & 1.15 & $0.76-1.74$ & 0.510 & $\mathrm{R}$ & $<0.001$ & 88.0 & & \\
\hline XX vs. XX & & 1.56 & $0.56-4.39$ & 0.399 & $\mathrm{R}$ & $<0.001$ & 88.9 & & \\
\hline$X x$ vs. XX & & 1.13 & $0.76-1.67$ & 0.540 & $\mathrm{R}$ & 0.021 & 59.8 & & \\
\hline$X x / X X$ vs. $x X$ & & 1.24 & $0.76-2.01$ & 0.387 & $\mathrm{R}$ & $<0.001$ & 76.2 & & \\
\hline$X X$ vs. $X x / x X$ & & 1.30 & $0.56-3.03$ & 0.536 & $\mathrm{R}$ & $<0.001$ & 88.2 & & \\
\hline Asian & 7 & & & & & & & & \\
\hline$X$ vs. X & & 1.23 & $0.47-3.25$ & 0.668 & R & $<0.001$ & 98.0 & & \\
\hline$X X$ vs. XX & & 2.18 & $0.37-12.73$ & 0.388 & R & $<0.001$ & 98.1 & & \\
\hline XX vs. XX & & 1.22 & $0.63-2.36$ & 0.553 & $\mathrm{R}$ & $<0.001$ & 88.0 & & \\
\hline$X x / X X$ vs. $X X$ & & 1.39 & $0.56-3.46$ & 0.481 & $\mathrm{R}$ & $<0.001$ & 94.6 & & \\
\hline$X X$ vs. $X x / x X$ & & 1.77 & $0.44-7.14$ & 0.424 & $\mathrm{R}$ & $<0.001$ & 96.0 & & \\
\hline \multicolumn{10}{|l|}{ ERa Pvull } \\
\hline Overall & 16 & & & & & & & & \\
\hline P vs. $p$ & & 0.96 & $0.71-1.29$ & 0.769 & $\mathrm{R}$ & $<0.001$ & 92.3 & 0.753 & 0.616 \\
\hline PP vs. pp & & 0.99 & $0.55-1.78$ & 0.961 & $\mathrm{R}$ & $<0.001$ & 90.8 & 1.000 & 0.886 \\
\hline Pp vs. pp & & 1.01 & $0.72-1.41$ & 0.956 & R & $<0.001$ & 82.3 & 0.753 & 0.501 \\
\hline PP/Pp vs. pp & & 0.97 & $0.65-1.43$ & 0.868 & $\mathrm{R}$ & $<0.001$ & 88.7 & 0.893 & 0.539 \\
\hline PP vs. Pp/pp & & 0.99 & $0.65-1.53$ & 0.977 & $\mathrm{R}$ & $<0.001$ & 87.3 & 0.893 & 0.976 \\
\hline Caucasian & 8 & & & & & & & & \\
\hline P vs. p & & 0.95 & $0.71-1.26$ & 0.716 & $\mathrm{R}$ & $<0.001$ & 79.2 & & \\
\hline PP vs. pp & & 0.93 & $0.49-1.79$ & 0.831 & $\mathrm{R}$ & $<0.001$ & 81.4 & & \\
\hline Pp vs. pp & & 0.98 & $0.73-1.31$ & 0.877 & $\mathrm{R}$ & 0.112 & 40.0 & & \\
\hline PP/Pp vs. pp & & 0.97 & $0.67-1.39$ & 0.861 & $\mathrm{R}$ & 0.008 & 63.5 & & \\
\hline PP vs. Pp/pp & & 0.97 & $0.59-1.58$ & 0.895 & $\mathrm{R}$ & $<0.001$ & 78.2 & & \\
\hline Asian & 8 & & & & & & & & \\
\hline P vs. $p$ & & 0.97 & $0.57-1.66$ & 0.919 & $\mathrm{R}$ & $<0.001$ & 95.6 & & \\
\hline PP vs. pp & & 1.08 & $0.40-2.96$ & 0.877 & $\mathrm{R}$ & $<0.001$ & 94.4 & & \\
\hline Pp vs. pp & & 1.04 & $0.58-1.88$ & 0.889 & $\mathrm{R}$ & $<0.001$ & 90.2 & & \\
\hline PP/Pp vs. pp & & 0.98 & $0.50-1.95$ & 0.962 & $\mathrm{R}$ & $<0.001$ & 93.8 & & \\
\hline PP vs. Pp/pp & & 1.05 & $0.50-2.20$ & 0.891 & $\mathrm{R}$ & $<0.001$ & 91.8 & & \\
\hline \multicolumn{10}{|l|}{ ERa G2014A } \\
\hline Overall & 4 & & & & & & & & \\
\hline A vs. G & & 0.89 & $0.32-2.51$ & 0.825 & $\mathrm{R}$ & $<0.001$ & 95.1 & 0.308 & 0.237 \\
\hline
\end{tabular}


Table 4 Results of genetic models for ERa Xbal, ERa Pvull, ERa G2014A, ERß Alul and ER $\beta$ Rsal polymorphisms and osteoporosis susceptibility in postmenopausal women (Continued)

\begin{tabular}{|c|c|c|c|c|c|c|c|c|c|}
\hline \multirow[t]{2}{*}{ Comparison } & \multirow[t]{2}{*}{$\mathrm{N}$} & \multicolumn{3}{|c|}{ Test of association } & \multirow[t]{2}{*}{ Model } & \multicolumn{2}{|c|}{ Test of heterogeneity } & \multirow{2}{*}{$\begin{array}{l}\text { Begg's test } \\
P \text { value }\end{array}$} & \multirow{2}{*}{$\begin{array}{l}\text { Egger's test } \\
P \text { value }\end{array}$} \\
\hline & & $\overline{\mathrm{OR}}$ & $95 \% \mathrm{Cl}$ & $P$ value & & $P$ value & $I^{2}(\%)$ & & \\
\hline AA vs. GG & & 0.88 & $0.08-9.19$ & 0.912 & $\mathrm{R}$ & $<0.001$ & 92.9 & 0.734 & 0.419 \\
\hline GA vs. GG & & 0.76 & $0.28-2.03$ & 0.581 & R & $<0.001$ & 88.1 & 0.734 & 0.530 \\
\hline GA/AA vs. GG & & 0.73 & $0.22-2.41$ & 0.601 & $\mathrm{R}$ & $<0.001$ & 92.8 & 0.734 & 0.530 \\
\hline AA vs. GA/GG & & 1.13 & $0.23-5.72$ & 0.878 & $\mathrm{R}$ & $<0.001$ & 88.6 & 0.734 & 0.299 \\
\hline Caucasian & 1 & & & & & & & & \\
\hline A vs. G & & 0.63 & $0.41-0.96$ & 0.032 & $\mathrm{R}$ & - & - & & \\
\hline AA vs. GG & & 0.28 & $0.07-1.21$ & 0.089 & $\mathrm{R}$ & - & - & & \\
\hline GA vs. GG & & 0.66 & $0.39-1.11$ & 0.116 & $\mathrm{R}$ & - & - & & \\
\hline GA/AA vs. GG & & 0.60 & $0.36-1.00$ & 0.050 & $\mathrm{R}$ & - & - & & \\
\hline AA vs. GA/GG & & 0.34 & $0.08-1.43$ & 0.141 & $\mathrm{R}$ & - & - & & \\
\hline Asian & 3 & & & & & & & & \\
\hline A vs. G & & 1.00 & $0.23-4.46$ & 0.996 & $\mathrm{R}$ & $<0.001$ & 96.6 & & \\
\hline AA vs. GG & & 1.28 & $0.05-30.10$ & 0.878 & R & $<0.001$ & 95.2 & & \\
\hline GA vs. GG & & 0.77 & $0.17-3.45$ & 0.736 & R & $<0.001$ & 91.3 & & \\
\hline GA/AA vs. GG & & 0.76 & $0.12-4.62$ & 0.765 & R & $<0.001$ & 94.8 & & \\
\hline AA vs. GA/GG & & 1.69 & $0.20-14.27$ & 0.630 & R & $<0.001$ & 92.2 & & \\
\hline \multicolumn{10}{|l|}{ ERß Alul } \\
\hline Overall & 4 & & & & & & & & \\
\hline A vs. G & & 1.25 & $0.78-2.00$ & 0.362 & R & $<0.001$ & 91.5 & 1.000 & 0.997 \\
\hline AA vs. GG & & 1.27 & $0.52-3.13$ & 0.597 & R & $<0.001$ & 88.4 & 0.734 & 0.647 \\
\hline GA vs. GG & & 1.16 & $0.65-2.07$ & 0.606 & R & 0.001 & 81.0 & 0.734 & 0.408 \\
\hline GA/AA vs. GG & & 1.29 & $0.66-2.53$ & 0.459 & $\mathrm{R}$ & $<0.001$ & 87.8 & 0.734 & 0.612 \\
\hline AA vs. GA/GG & & 1.21 & $0.65-2.24$ & 0.553 & R & $<0.001$ & 85.7 & 0.497 & 0.646 \\
\hline Caucasian & 3 & & & & & & & & \\
\hline A vs. G & & 1.23 & $0.58-2.57$ & 0.590 & $\mathrm{R}$ & $<0.001$ & 94.3 & & \\
\hline AA vs. GG & & 1.28 & $0.34-4.84$ & 0.717 & $\mathrm{R}$ & $<0.001$ & 92.2 & & \\
\hline GA vs. GG & & 1.30 & $0.60-2.78$ & 0.504 & R & 0.001 & 86.5 & & \\
\hline GA/AA vs. GG & & 1.36 & $0.55-3.39$ & 0.507 & $\mathrm{R}$ & $<0.001$ & 91.8 & & \\
\hline AA vs. GA/GG & & 1.10 & $0.37-3.22$ & 0.863 & R & $<0.001$ & 90.3 & & \\
\hline Asian & 1 & & & & & & & & \\
\hline A vs. G & & 1.31 & $1.06-1.62$ & 0.012 & R & - & - & & \\
\hline AA vs. GG & & 1.24 & $0.72-2.13$ & 0.441 & $\mathrm{R}$ & - & - & & \\
\hline GA vs. GG & & 0.84 & $0.48-1.48$ & 0.548 & $\mathrm{R}$ & - & - & & \\
\hline GA/AA vs. GG & & 1.10 & $0.64-1.87$ & 0.739 & R & - & - & & \\
\hline AA vs. GA/GG & & 1.44 & $1.12-1.84$ & 0.004 & $\mathrm{R}$ & - & - & & \\
\hline \multicolumn{10}{|l|}{ ER $\beta$ Rsal } \\
\hline Overall & 2 & & & & & & & & \\
\hline A vs. G & & 0.92 & $0.50-1.70$ & 0.785 & $\mathrm{R}$ & 0.010 & 85.0 & & \\
\hline AA vs. GG & & 0.49 & $0.34-0.70$ & $<0.001$ & $\mathrm{~F}$ & 0.261 & 20.9 & & \\
\hline GA vs. GG & & 0.87 & $0.41-1.84$ & 0.722 & R & $<0.001$ & 85.9 & & \\
\hline GA/AA vs. GG & & 0.85 & $0.37-1.95$ & 0.704 & $\mathrm{R}$ & $<0.001$ & 88.9 & & \\
\hline
\end{tabular}


Table 4 Results of genetic models for ERa Xbal, ERa Pvull, ERa G2014A, ER $\beta$ Alul and ER $\beta$ Rsal polymorphisms and osteoporosis susceptibility in postmenopausal women (Continued)

\begin{tabular}{|c|c|c|c|c|c|c|c|c|c|}
\hline \multirow[t]{2}{*}{ Comparison } & \multirow[t]{2}{*}{$\mathrm{N}$} & \multicolumn{3}{|c|}{ Test of association } & \multirow[t]{2}{*}{ Model } & \multicolumn{2}{|c|}{ Test of heterogeneity } & \multirow{2}{*}{$\begin{array}{l}\text { Begg's test } \\
P \text { value }\end{array}$} & \multirow{2}{*}{$\begin{array}{l}\text { Egger's test } \\
P \text { value }\end{array}$} \\
\hline & & $\overline{\mathrm{OR}}$ & $95 \% \mathrm{Cl}$ & $P$ value & & $P$ value & $1^{2}(\%)$ & & \\
\hline AA vs. GA/GG & & 0.66 & $0.48-0.90$ & 0.009 & $\mathrm{~F}$ & 0.408 & 0 & & \\
\hline Caucasian & 1 & & & & & & & & \\
\hline A vs. G & & 1.30 & $0.83-2.04$ & 0.245 & $\mathrm{R}$ & - & - & & \\
\hline AA vs. GG & & 1.92 & $0.17-21.41$ & 0.596 & $\mathrm{~F}$ & - & - & & \\
\hline GA vs. GG & & 1.32 & $0.80-2.15$ & 0.273 & $\mathrm{R}$ & - & - & & \\
\hline GA/AA vs. GG & & 1.33 & $0.82-2.17$ & 0.246 & $\mathrm{R}$ & - & - & & \\
\hline AA vs. GA/GG & & 1.81 & $0.16-20.11$ & 0.630 & $\mathrm{~F}$ & - & - & & \\
\hline Asian & 1 & & & & & & & & \\
\hline A vs. G & & 0.69 & $0.58-0.82$ & $<0.001$ & $\mathrm{R}$ & - & - & & \\
\hline AA vs. GG & & 0.47 & $0.33-0.68$ & $<0.001$ & $\mathrm{~F}$ & - & - & & \\
\hline GA vs. GG & & 0.61 & $0.47-0.81$ & $<0.001$ & $\mathrm{R}$ & - & - & & \\
\hline GA/AA vs. GG & & 0.57 & $0.44-0.74$ & $<0.001$ & $\mathrm{R}$ & - & - & & \\
\hline AA vs. GA/GG & & 0.65 & $0.47-0.89$ & 0.007 & $\mathrm{~F}$ & - & - & & \\
\hline
\end{tabular}

$R$ Random effect model

$F$ Fixed effect model

mediating estrogen-induced repression of TNF- $\alpha$ expression, which is considered an important contributor to PMOP [30]. ER $\beta$ AluI is one of the widelystudied ER $\beta$ gene polymorphisms, knowing that it could alter mRNA stability and protein levels, leading to reduced synthesis of ER $\beta$ [30]. In our study, ER $\beta$ AluI was found to be significantly associated with increased risk of PMOP in Asian populations, while no significant relationship was observed in overall and Caucasian populations. Thus, different genetic backgrounds, environmental effects and/or their internal interactions could explain the diverse results in various ethnicities. ER $\beta$ RsaI is another important polymorphism of ER $\beta$. Our subgroup analysis revealed a significant association between ER $\beta$ Rs $a \mathrm{I}$ and PMOP risk in overall populations, which is consistent with the studies of Shoukry et al. [30], and Huang et al. [31].

\section{Associations between ERa Xbal and lumbar spine and femoral neck BMD}

Our pooled results showed that there was no significant difference in lumbar spine BMD between PMOP women carrying $\mathrm{XX}, \mathrm{Xx}$ and $\mathrm{xx}$ genotype in either overall, Caucasian or Asian populations. However, WANG et al. [39] reported that the XbaI

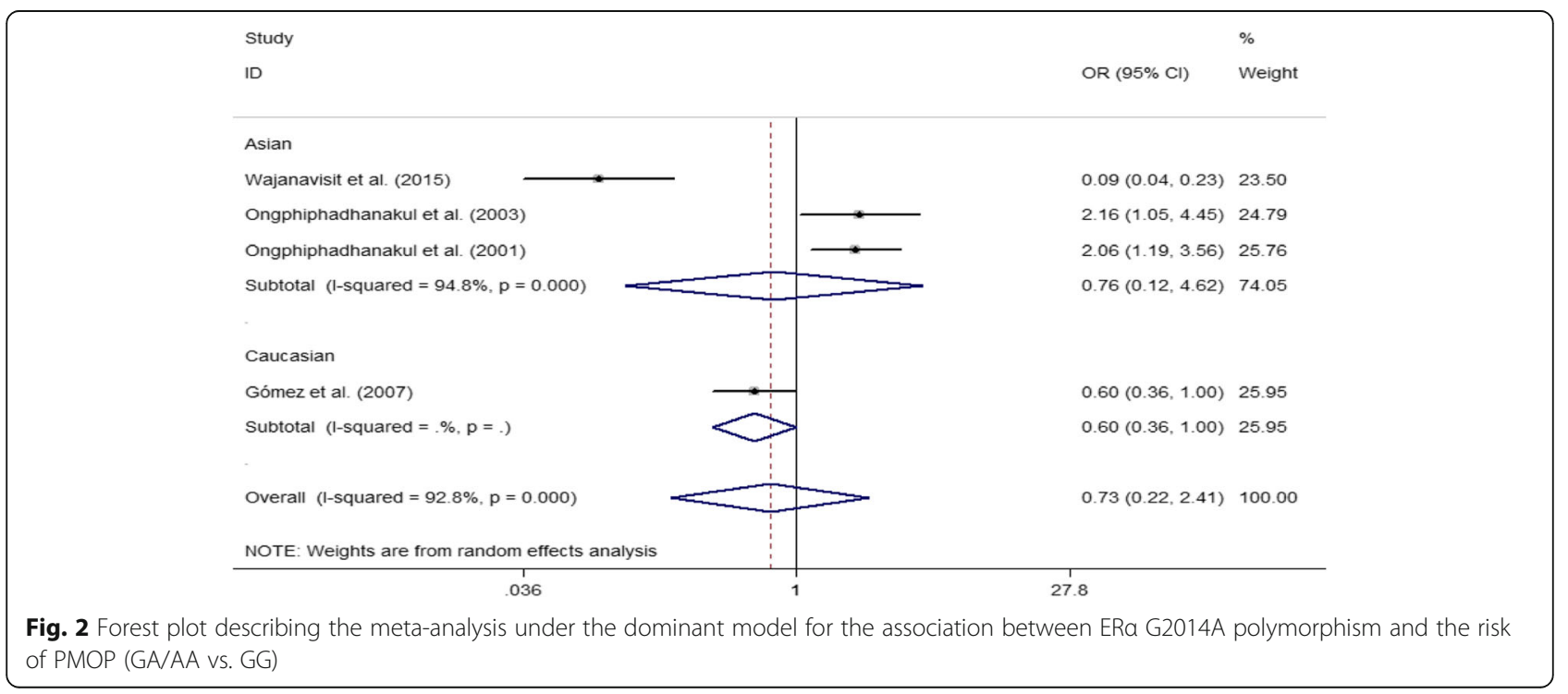




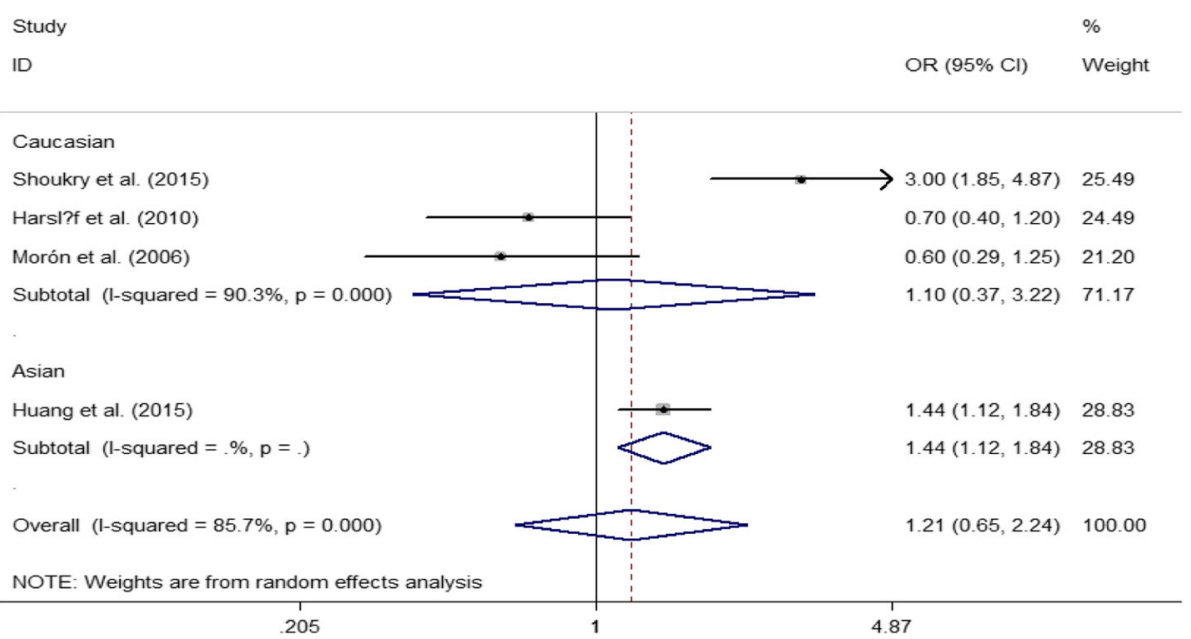

Fig. 3 Forest plot describing the meta-analysis under the recessive model for the association between ER $\beta$ Alul polymorphism and the risk of PMOP (AA vs. GA/GG)

polymorphism was significantly associated with BMD of the lumbar spine, and XX had a protective effect in comparison with carriers of the $\mathrm{x}$ alleles, which is consistent with the report of Ioannidis et al. [41]. Both WANG and Ioannidis included all types of osteoporotic patients, not only postmenopausal women, which might be the most important reason for the difference between our results and theirs. As mentioned above, $\mathrm{ER} \alpha \mathrm{XbaI}$ might not play a key role in attainment and maintenance of peek bone mass in postmenopausal women [24], and therefore it could be easily understood why no significant association was observed between ER $\alpha$ XbaI and lumbar spine BMD. With regard to femoral neck BMD, our study indicated that the femoral neck BMD in PMOP women with XX genotype was significantly higher than that in women with $\mathrm{xx}$ genotype in overall and Caucasian populations, which highlights the theory that ER $\alpha$ gene is involved in the pathogenesis of PMOP. No significant difference of femoral neck BMD was observed between PMOP women with $\mathrm{Xx}$ and $\mathrm{xx}$ genotype in each subgroup. Although no significant association was observed between lumbar spine BMD and ER $\alpha b a \mathrm{I}$, we found that the lumbar spine $\mathrm{Z}$ value in both PMOP women carrying $\mathrm{XX}$ and those carrying $\mathrm{Xx}$ genotype was significantly higher than that in Caucasians carrying $\mathrm{xx}$ genotype. We also observed that XX genotype was associated with a low femoral neck $Z$ value in Caucasians and high femoral neck $Z$ value in Asians. In addition, Caucasians and Asians carrying $\mathrm{Xx}$ genotype were at risk of a high femoral neck $\mathrm{Z}$ value. However, why ER $\alpha$ XbaI plays a contradictory role in BMD and $\mathrm{Z}$ value at the lumar spine and femoral neck, and the mechanisms by which it is associated with BMD and $\mathrm{Z}$ value remains unclear and needs further investigation.

\section{Associations between ERa Pvull and lumbar spine and femoral neck BMD}

Although the molecular mechanism underlying the effect of ER $\alpha$ PvuII on bone mass is poorly understood, it is believed that ER $\alpha$ PvuII might play a key role in BMD as it is in linkage disequilibrium with the TA polymorphism in the ER promoter that is associated with altered gene transcription [20]. Our pooled analysis indicated that PMOP women with the Pp genotype had lower lumbar spine BMD than those with the pp. genotype. We also found that there was no significant difference in lumbar spine BMD between women with the PP genotype and those with the pp. genotype, which is consistent with the meta-analysis of Wang et al. [40]. Furthermore, we observed that the PP genotype was associated with decreased femoral neck BMD in Asians, while Pp might not play a key role in femoral neck BMD in all subgroups. Interestingly, WANG et al. [39] reported that PP play a role in protecting the lumbar spine but on the other hand it might be a risk factor for the femoral neck fracture. Wang CL [40] and WANG KJ [39] conducted their meta-analyses on osteoporotic women during menopause while our study included osteoporotic women post menopause, which might be the most important reason for the difference between our study and theirs. In addition, both PP and Pp genotypes were significantly associated with low lumbar spine $\mathrm{Z}$ value in Caucasians, 
Table 5 Meta-analysis of differences of Lumbar Spine BMD, Femoral Neck BMD, Lumbar Spine Z value and Femoral Neck Z value between each genotype of ERa Xbal and ERa Pvull polymorphism

\begin{tabular}{|c|c|c|c|c|c|c|c|c|c|c|c|c|}
\hline \multirow[t]{3}{*}{ ERa Xbal } & \multicolumn{6}{|c|}{$X X$ vs. XX } & \multicolumn{6}{|c|}{ Xx vs. XX } \\
\hline & \multicolumn{3}{|c|}{ Test of differences } & \multirow[t]{2}{*}{ Model } & \multicolumn{2}{|c|}{$\begin{array}{l}\text { Test of } \\
\text { heterogeneity }\end{array}$} & \multicolumn{3}{|c|}{ Test of differences } & \multirow[t]{2}{*}{ Model } & \multicolumn{2}{|c|}{$\begin{array}{l}\text { Test of } \\
\text { heterogeneity }\end{array}$} \\
\hline & $\mathrm{N}$ & WMD (95\% Cl) & $P$ value & & $P$ value & $I^{2}(\%)$ & $\mathrm{N}$ & WMD (95\% Cl) & $P$ value & & $P$ value & $I^{2}(\%)$ \\
\hline \multicolumn{13}{|c|}{ Lumbar Spine BMD $\left(\mathrm{g} / \mathrm{cm}^{2}\right)$} \\
\hline Overall & 8 & $0.03(-0.02,0.08)$ & 0.198 & $\mathrm{R}$ & $<0.001$ & 94.2 & 8 & $0.02(-0.00,0.05)$ & 0.086 & R & $<0.001$ & 94.1 \\
\hline Caucasian & 5 & $0.00(-0.04,0.04)$ & 0.917 & $\mathrm{R}$ & $<0.001$ & 90.2 & 5 & $0.00(-0.02,0.02)$ & 0.862 & $\mathrm{R}$ & $<0.001$ & 91.1 \\
\hline Asian & 3 & $0.11(-0.16,0.38)$ & 0.414 & $\mathrm{R}$ & $<0.001$ & 97.8 & 3 & $0.07(-0.07,0.20)$ & 0.326 & $R$ & $<0.001$ & 97.3 \\
\hline \multicolumn{13}{|c|}{ Lumbar Spine $Z$ value } \\
\hline Overall & 3 & $0.22(-0.40,0.83)$ & 0.495 & $\mathrm{R}$ & $<0.001$ & 88.5 & 3 & $0.24(0.00,0.47)$ & 0.046 & $R$ & 0.041 & 68.6 \\
\hline Caucasian & 1 & $0.13(0.05,0.21)$ & 0.001 & R & - & - & 1 & $0.18(0.15,0.21)$ & $<0.001$ & $R$ & - & - \\
\hline Asian & 2 & $-0.28(-2.58,2.02)$ & 0.811 & $\mathrm{R}$ & 0.009 & 85.2 & 2 & $-0.23(-1.81,1.36)$ & 0.780 & $\mathrm{R}$ & 0.062 & 71.3 \\
\hline \multicolumn{13}{|c|}{ Femoral Neck BMD $\left(\mathrm{g} / \mathrm{cm}^{2}\right)$} \\
\hline Overall & 6 & $0.03(0.01,0.05)$ & 0.003 & $\mathrm{R}$ & 0.001 & 75.5 & 6 & $0.01(-0.00,0.03)$ & 0.057 & $R$ & $<0.001$ & 84.7 \\
\hline Caucasian & 5 & $0.03(0.01,0.05)$ & 0.009 & R & $<0.001$ & 80.4 & 5 & $0.01(-0.00,0.03)$ & 0.094 & R & $<0.001$ & 87.7 \\
\hline Asian & 1 & $0.03(-0.01,0.08)$ & 0.110 & $\mathrm{R}$ & - & - & 1 & $0.01(-0.02,0.04)$ & 0.350 & R & - & - \\
\hline \multicolumn{13}{|c|}{ Femoral Neck Z value } \\
\hline Overall & 2 & $-0.38(-2.56,1.80)$ & 0.733 & $\mathrm{R}$ & $<0.001$ & 99.2 & 2 & $0.25(-0.07,0.58)$ & 0.130 & R & 0.001 & 91.6 \\
\hline Caucasian & 1 & $-1.48(-1.57,-1.39)$ & $<0.001$ & $\mathrm{R}$ & - & - & 1 & $0.10(0.07,0.13)$ & $<0.001$ & R & - & - \\
\hline Asian & 1 & $0.74(0.37,1.11)$ & $<0.001$ & $\mathrm{R}$ & - & - & 1 & $0.43(0.24,0.62)$ & $<0.001$ & R & - & - \\
\hline \multirow[t]{3}{*}{ ERa Pvull } & \multicolumn{6}{|c|}{ PP vs. pp } & \multicolumn{6}{|c|}{ Pp vs. pp } \\
\hline & \multicolumn{3}{|c|}{ Test of differences } & Model & \multicolumn{2}{|c|}{$\begin{array}{l}\text { Test of } \\
\text { heterogeneity }\end{array}$} & \multicolumn{3}{|c|}{ Test of differences } & Model & \multicolumn{2}{|c|}{$\begin{array}{l}\text { Test of } \\
\text { heterogeneity }\end{array}$} \\
\hline & $\mathrm{N}$ & WMD (95\% Cl) & $P$ value & & $P$ value & $1^{2}(\%)$ & $\mathrm{N}$ & WMD (95\% Cl) & $P$ value & & $P$ value & $P^{2}(\%$ \\
\hline \multicolumn{13}{|c|}{ Lumbar Spine BMD $\left(\mathrm{g} / \mathrm{cm}^{2}\right)$} \\
\hline Overall & 10 & $0.02(-0.01,0.04)$ & 0.216 & $\mathrm{R}$ & $<0.001$ & 95.5 & 10 & $-0.01(-0.02,-0.00)$ & 0.036 & $R$ & $<0.001$ & 84.0 \\
\hline Caucasian & 5 & $0.01(-0.04,0.06)$ & 0.793 & $\mathrm{R}$ & $<0.001$ & 95.5 & 5 & $-0.02(-0.03,0.00)$ & 0.106 & R & $<0.001$ & 84.9 \\
\hline Asian & 5 & $0.03(-0.02,0.08)$ & 0.288 & $\mathrm{R}$ & $<0.001$ & 96.2 & 5 & $-0.00(-0.02,0.02)$ & 0.912 & $\mathrm{R}$ & $<0.001$ & 86.4 \\
\hline \multicolumn{13}{|c|}{ Lumbar Spine Z value } \\
\hline Overall & 3 & $0.11(-0.55,0.78)$ & 0.742 & $\mathrm{R}$ & $<0.001$ & 98.7 & 3 & $0.13(-0.40,0.67)$ & 0.623 & R & $<0.001$ & 95.9 \\
\hline Caucasian & 1 & $-0.07(-0.13,-0.01)$ & 0.031 & $\mathrm{R}$ & - & - & 1 & $-0.16(-0.20,-0.12)$ & $<0.001$ & R & - & - \\
\hline Asian & 2 & $0.24(-1.72,2.20)$ & 0.809 & R & $<0.001$ & 99.0 & 2 & $0.34(-1.18,1.85)$ & 0.665 & $\mathrm{R}$ & $<0.001$ & 97.9 \\
\hline \multicolumn{13}{|c|}{ Femoral Neck BMD $\left(\mathrm{g} / \mathrm{cm}^{2}\right)$} \\
\hline Overall & 8 & $-0.04(-0.09,0.01)$ & 0.135 & $\mathrm{R}$ & $<0.001$ & 99.3 & 8 & $-0.02(-0.04,0.01)$ & 0.132 & $R$ & $<0.001$ & 98.2 \\
\hline Caucasian & 5 & $-0.06(-0.16,0.05)$ & 0.295 & $\mathrm{R}$ & $<0.001$ & 99.6 & 5 & $-0.03(-0.05,0.00)$ & 0.054 & $\mathrm{R}$ & $<0.001$ & 95.2 \\
\hline Asian & 3 & $-0.01(-0.02,-0.01)$ & $<0.001$ & $\mathrm{R}$ & 1.000 & 0.00 & 3 & $-0.00(-0.03,0.02)$ & 0.768 & $\mathrm{R}$ & 0.009 & 78.7 \\
\hline \multicolumn{13}{|c|}{ Femoral Neck Z value } \\
\hline Overall & 2 & $-0.39(-1.15,0.37)$ & 0.315 & R & $<0.001$ & 97.0 & 2 & $-0.39(-0.57,-0.20)$ & $<0.001$ & $R$ & 0.024 & 80.3 \\
\hline Caucasian & 1 & $-0.01(-0.08,0.05)$ & 0.718 & R & - & - & 1 & $-0.31(-0.35,-0.27)$ & $<0.001$ & R & - & - \\
\hline Asian & 1 & $-0.79(-1.05,-0.53)$ & $<0.001$ & $\mathrm{R}$ & - & - & 1 & $-0.50(-0.66,-0.34)$ & $<0.001$ & $R$ & - & - \\
\hline
\end{tabular}

$R$ Random effect model

$F$ Fixed effect model

but not in overall and Asian populations, probably because of the different genetic backgrounds in various ethnicities and interactions between genetic and non-genetic factors. PMOP women with the PP and Pp genotypes had lower femoral neck $\mathrm{Z}$ value than those with the pp. genotype in overall, Caucasian and Asian populations. 


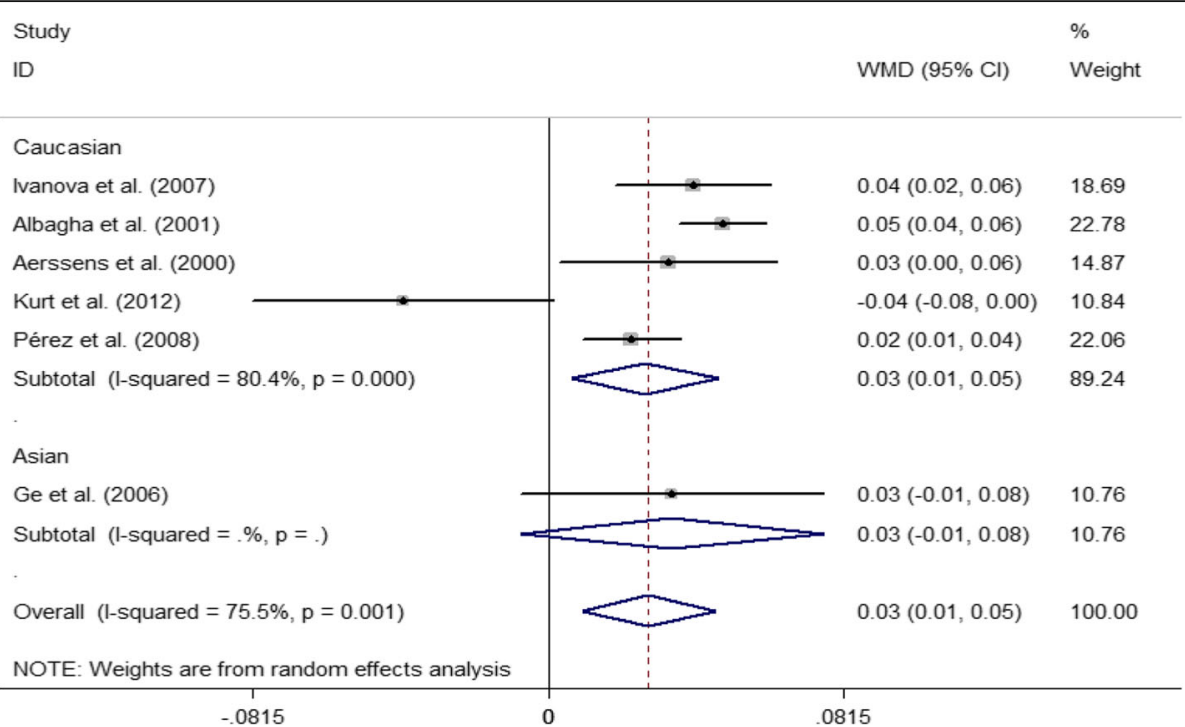

Fig. 4 Forest plot showed that XX genotype of ERa Xbal was associated with increased femoral neck BMD compared with XX genotype

\section{Limitations}

Although we performed a comprehensive analysis of the association between ER $\alpha, \operatorname{ER} \beta$ gene polymorphisms and PMOP risk and BMD in postmenopausal women, there are some limitations that should be addressed. First, high heterogeneity was observed in some of our pooled results, which might have negative impact on our conclusions. Second, PMOP is a disease whose etiology might be involved in several confounding factors, and other confounding factors such as age, years since menopause and estrogen therapy might interact with each other and play a key role in the etiology and progression of PMOP. However, no data available could be used in all recruited studies to detect the interactions between these confounding factors in PMOP patients. We should take all these confounding factors into consideration in our study rather than studying them separately, which is also a limitation of our meta-analysis. Third, we failed to perform a pooled analysis to detect whether ER $\alpha$ G2014A, ER $\beta$ AluI and ER $\beta$ RsaI were correlated with BMD in postmenopausal women as no sufficient data could be collected and analyzed. Therefore, largerscale and better-designed studies are necessary to determine the association between $E R \alpha / \beta$ gene polymorphisms and PMOP risk and BMD in postmenopausal women.

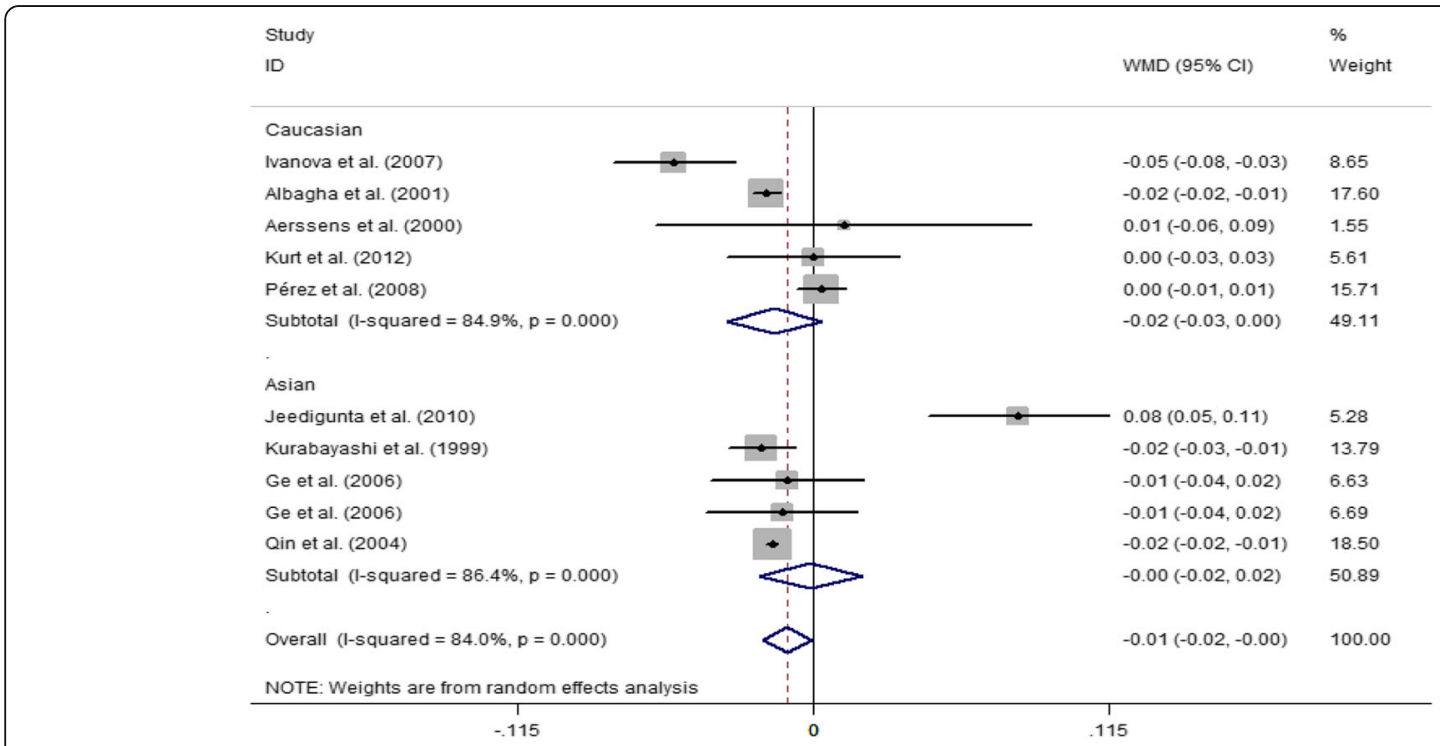

Fig. 5 Forest plot showed that Pp genotype of ERa Pvull was associated with increased lumbar spine BMD compared with pp. genotype 


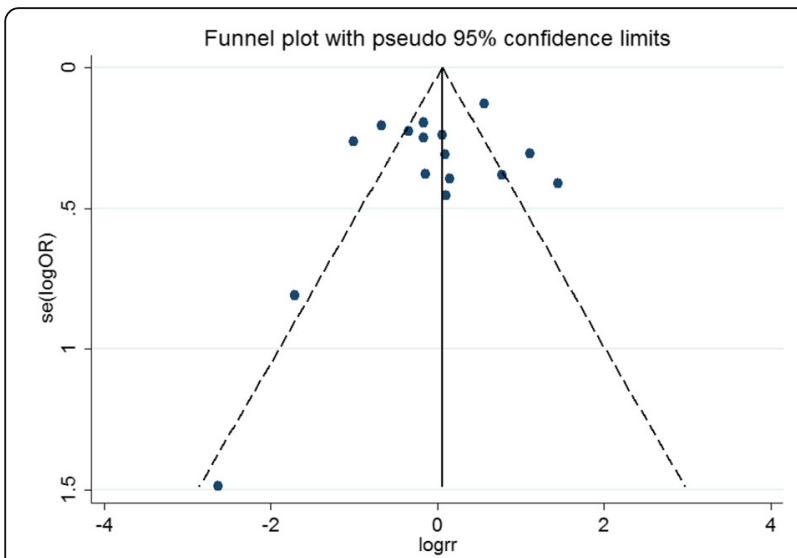

Fig. 6 Funnel plot of the ERa Pvull polymorphism and PMOP risk

\section{Conclusion}

$E R \alpha / \beta$ gene polymorphisms were significantly associated with PMOP risk and BMD in postmenopausal women, but each $E R \alpha / \beta$ gene polymorphism may have a distinct effect on PMOP risk and BMD in Asian and Caucasian populations.

\section{Abbreviations}

BMD: Bone mineral density; Cl: Confidence interval; ER: Estrogen receptor; Lactase: LCT; OR: Odds ratios; PMOP: Postmenopausal Osteoporosis; PTH: Parathyroid Hormone; TGF- $\beta$ : Transforming growth factor- $\beta$; WMD: Weight mean difference

\section{Acknowledgements}

Not applicable

\section{Funding}

No

\section{Availability of data and materials}

In publicly available repositories

\section{Authors' contributions}

HPZ and JNJ participated in the study design. QW and JZ made contributions to the data collection. LZ, YJX and TLM were responsible for the statistical analysis. HPZ and LYZ participated in the writting and LYZ was also responsible for the final proofing. All authors read and approved the final manuscript.

\section{Ethics approval and consent to participate}

Not applicable

\section{Consent for publication}

Not applicable

\section{Competing interests}

The authors declare that they have no competing interests

\section{Publisher's Note}

Springer Nature remains neutral with regard to jurisdictional claims in published maps and institutional affiliations.

\section{Author details}

${ }^{1}$ Department of Orthopedics, The Affiliated Yixing Hospital of Jiangsu University, Yixing 214200, China. ${ }^{2}$ Department of Orthopedics, The Second Affiliated Hospital of Soochow University, Suzhou 215004, China.

${ }^{3}$ Department of Orthopedics, Northern Jiangsu People's Hospital, Yangzhou
225001, China. ${ }^{4}$ Central Laboratory, The Affiliated Yixing Hospital of Jiangsu University, Yixing 214200, China.

Received: 13 June 2017 Accepted: 17 January 2018

Published online: 20 February 2018

\section{References}

1. Gokosmanoglu F, Varim C, Atmaca A, Atmaca MH, Colak R. The effects of zoledronic acid treatment on depression and quality of life in women with postmenopausal osteoporosis: a clinical trial study. Journal of research in medical sciences : the official journal of Isfahan University of Medical Sciences. 2016;21:112.

2. Bandeira L, Bilezikian JP. Novel therapies for postmenopausal osteoporosis. Endocrinol Metab Clin N Am. 2017;46(1):207-19.

3. Eastell R, O'Neill TW, Hofbauer LC, Langdahl B, Reid IR, Gold DT, Cummings SR. Postmenopausal osteoporosis. Nature reviews Disease primers. 2016;2: 16069.

4. Wensel TM, Iranikhah MM, Wilborn TW. Effects of denosumab on bone mineral density and bone turnover in postmenopausal women. Pharmacotherapy. 2011;31(5):510-23.

5. Paschalis EP, Gamsjaeger S, Hassler N, Fahrleitner-Pammer A, Dobnig H, Stepan JJ, Pavo I, Eriksen EF, Klaushofer K. Vitamin D and calcium supplementation for three years in postmenopausal osteoporosis significantly alters bone mineral and organic matrix quality. Bone. 2017;95: 41-6.

6. Ebina K, Kashii M, Hirao M, Hashimoto J, Noguchi T, Koizumi K, Kitaguchi K, Matsuoka H, Iwahashi T, Tsukamoto Y, et al. Comparison of the effects of denosumab between a native vitamin $\mathrm{D}$ combination and an active vitamin D combination in patients with postmenopausal osteoporosis. J Bone Miner Metab. 2016;

7. Safer U, Safer VB, Demir SO, Yanikoglu I. Effects of bisphosphonates and calcium plus vitamin-D supplements on cognitive function in postmenopausal osteoporosis section sign. Endocrine, metabolic \& immune disorders drug targets. 2016;16(1):56-60

8. Gennari L, Rotatori S, Bianciardi S, Nuti R, Merlotti D. Treatment needs and current options for postmenopausal osteoporosis. Expert Opin Pharmacother. 2016;17(8):1141-52.

9. Xie W, Ji L, Zhao T, Gao P. Identification of transcriptional factors and key genes in primary osteoporosis by DNA microarray. Medical science monitor international medical journal of experimental and clinical research. 2015;21: 1333-44.

10. Macari S, Ajay Sharma L, Wyatt A, Knowles P, Szawka RE, Garlet GP, Grattan DR, Dias GJ, Silva TA. Osteoprotective effects of estrogen in the maxillary bone depend on ERalpha. J Dent Res. 2016;95(6):689-96.

11. Shang DP, Lian HY, Fu DP, Wu J, Hou SS, Lu JM. Relationship between estrogen receptor 1 gene polymorphisms and postmenopausa osteoporosis of the spine in Chinese women. Genetics and molecular research : GMR. 2016;15(2)

12. Wang ZR. Age diference of estrogen receptor gene polymorphisms in the elderly women with hip osteoporosis. Chin. J.of Tissue Engmeenng Res. 2015;19(7):991-5.

13. Hai L, Jishen $X$, Bingpu $C$, Hailing $H$, Jinhua $W$, Jianhai $C$, xiaoyan $F$. Estrogen receptor- alpha gene Pvull, Xbal polymorphism, camellia oil and postmenopausal osteoporosis relevance in Guangxi Zhuang. Chinese. J Anat. 2014:37(5):581-4.

14. Erdogan MO, Yildiz H, Artan S, Solak M, Tascioglu F, Dundar U, Eser B, Colak E. Association of estrogen receptor alpha and collagen type I alpha 1 gene polymorphisms with bone mineral density in postmenopausal women. Osteoporosis international : a journal established as result of cooperation between the European Foundation for Osteoporosis and the National Osteoporosis Foundation of the USA. 2011;22(4):1219-25.

15. Jeedigunta Y, Bhoomi Reddy PR, Kolla VK, Munshi A, Ananthapur V, Narasimulu G, Akka J. Association of estrogen receptor alpha gene polymorphisms with BMD and their affect on estradiol levels in pre- and postmenopausal women in south Indian population from Andhra Pradesh. Clin. Chim. Acta ; Int. J. of clin. Chem. 2010:411(7-8):597-600.

16. Durusu Tanriover M, Bora Tatar G, Uluturk TD, Dayangac Erden D, Tanriover A, Kilicarslan A, Oz SG, Erdem Yurter H, Sozen T, Sain Guven G. Evaluation of the effects of vitamin $D$ receptor and estrogen receptor 1 gene polymorphisms on bone mineral density in postmenopausal women. Clin Rheumatol. 2010;29(11):1285-93. 
17. Harslof T, Husted LB, Carstens M, Stenkjaer L, Langdahl BL. Genotypes and haplotypes of the estrogen receptor genes, but not the retinoblastomainteracting zinc finger protein 1 gene, are associated with osteoporosis. Calcif Tissue Int. 2010;87(1):25-35.

18. Musumeci M, Vadala G, Tringali G, Insirello E, Roccazzello AM, Simpore J, Musumeci S. Genetic and environmental factors in human osteoporosis from sub-Saharan to Mediterranean areas. J Bone Miner Metab. 2009;27(4): 424-34.

19. Perez A, Ulla M, Garcia B, Lavezzo M, Elias E, Binci M, Rivoira M, Centeno V, Alisio A, Tolosa de Talamoni N. Genotypes and clinical aspects associated with bone mineral density in argentine postmenopausal women. J Bone Miner Metab. 2008;26(4):358-65.

20. Ivanova JT, Doukova PB, Boyanov MA, Popivanov PR. Pvull and Xbal polymorphisms of the estrogen receptor gene and bone mineral density in a Bulgarian population sample. Hormones (Athens, Greece). 2007;6(1):36-43.

21. Wang W, Fu SJ, Wang XS, Zhang YP, Wang SW, Zhong B. The relationship between ER gene polymorphisms and postmenopause osteoporosis of spine in southern Chinese women. Jurnal of Clinical Orthopsedics. 2006;9(6): 562-5.

22. Nam HS, Shin MH, Kweon SS, Park KS, Sohn SJ, Rhee JA, Choi JS, Son MH. Association of estrogen receptor-alpha gene polymorphisms with bone mineral density in postmenopausal Korean women. J Bone Miner Metab. 2005:23(1):84-9.

23. Qin YJ, Zhang ZL, Huang QR, He JW, Zhou Q, Hu YQ, Li M, Liu YJ. Association of ER alpha gene Pvull and Xbal polymorphisms and related factors with osteoporosis in postmenopausal women: a case-control study. Chin J Geriatr. 2004;23(6):380-3.

24. Aerssens J, Dequeker J, Peeters J, Breemans S, Broos P, Boonen S. Polymorphisms of the VDR, ER and COLIA1 genes and osteoporotic hip fracture in elderly postmenopausal women. Osteoporosis international : a journal established as result of cooperation between the European Foundation for Osteoporosis and the National Osteoporosis Foundation of the USA. 2000;11(7):583-91.

25. Sonoda T, Takada J, Iba K, Asakura S, Yamashita T, Mori M. Interaction between ESRa polymorphisms and environmental factors in osteoporosis. J Orthop Res. 2012:30(10):1529-34.

26. Wajanavisit W, Suppachokmongkorn S, Woratanarat $P$, Ongphiphadhanakul B, Tawonsawatruk T. The association of bone mineral density and G2014A polymorphism in the estrogen receptor alpha gene in osteoporotic hip fracture in Thai population. Journal of the Medical Association of Thailand = Chotmaihet thangphaet. 2015;98(Suppl 8):S82-7.

27. Gomez R, Magana JJ, Cisneros B, Perez-Salazar E, Faugeron S, Veliz D, Castro C, Rubio J, Casas L, Valdes-Flores M. Association of the estrogen receptor alpha gene polymorphisms with osteoporosis in the Mexican population. Clin Genet. 2007;72(6):574-81.

28. Ongphiphadhanakul B, Chanprasertyothin S, Payattikul P, Saetung S, Rajatanavin R. The implication of assessing a polymorphism in estrogen receptor alpha gene in the risk assessment of osteoporosis using a screening tool for osteoporosis in Asians. Osteoporosis international : a journal established as result of cooperation between the European Foundation for Osteoporosis and the National Osteoporosis Foundation of the USA. 2003;14(10):863-7.

29. Ongphiphadhanakul B, Chanprasertyothin S, Payattikul P, Saetung S, Piaseu N, Chailurkit L, Rajatanavin R. Association of a G2014A transition in exon 8 of the estrogen receptor-alpha gene with postmenopausal osteoporosis. Osteoporosis international : a journal established as result of cooperation between the European Foundation for Osteoporosis and the National Osteoporosis Foundation of the USA. 2001;12(12):1015-9.

30. Shoukry A, Shalaby SM, Etewa RL, Ahmed HS, Abdelrahman HM. Association of estrogen receptor beta and estrogen-related receptor alpha gene polymorphisms with bone mineral density in postmenopausal women. Mol Cell Biochem. 2015;405(1-2):23-31.

31. Huang HL, Tan HH, Chen BP, Li H, Xie JS, Zhao QZ. Correlation of polymorphism of estrogen receptor- $\beta$ and camellia oil with postmenopausal osteoporosis in Zhuang women of Guangxi. Chinese Journal of Anatomy. 2015;03:323-325,343.

32. Moron FJ, Mendoza N, Vazquez F, Molero E, Quereda F, Salinas A, Fontes J, Martinez-Astorquiza T, Sanchez-Borrego R, Ruiz A. Multilocus analysis of estrogen-related genes in Spanish postmenopausal women suggests an interactive role of ESR1, ESR2 and NRIP1 genes in the pathogenesis of osteoporosis. Bone. 2006;39(1):213-21.
33. Albagha OM, FE MG, Reid DM, Ralston SH. Estrogen receptor alpha gene polymorphisms and bone mineral density: haplotype analysis in women from the United Kingdom. Journal of bone and mineral research : the official journal of the American Society for Bone and Mineral Research. 2001; 16(1):128-34

34. Kurt O, Yilmaz-Aydogan H, Uyar M, Isbir T, Seyhan MF, Can A. Evaluation of ERalpha and VDR gene polymorphisms in relation to bone mineral density in Turkish postmenopausal women. Mol Biol Rep. 2012;39(6):6723-30.

35. Kurabayashi T, Tomita M, Matsushita H, Yahata T, Honda A, Takakuwa K, Tanaka K. Association of vitamin D and estrogen receptor gene polymorphism with the effect of hormone replacement therapy on bone mineral density in Japanese women. Am J Obstet Gynecol. 1999;180(5): $1115-20$.

36. Ge JR, Zhu XX, Chen K. Effect of estrogen receptor gene Px haplotype on bone mineral density in female postmenopausal osteoporosis. Chin J Geriatr. 2006;25(6):416-9.

37. Ge JR, Wang HM, Zhu XX, Chen K. Effect of Pvullpolymorphisms of estrogen receptor gene on filtering risk factors in postmenopansal osteoporosis. Chin J Osteoporos. 2006;12(1):38-40.

38. An SJ, Li E, Tong XX, Liu K, Zhao JS. Study on relationship between estrogen receptor gene polymorphism and syndrome differentiation typing of female postmenopausal osteoporosis in traditional Chinese medicine. Chinese Journal of Integrated Traditional and Western Medicine. 2000; 20(12):907-10.

39. Wang KJ, Shi DQ, Sun LS, Jiang X, Lu YY, Dai J, Chen DY, Xu ZH, Jiang Q. Association of estrogen receptor alpha gene polymorphisms with bone mineral density: a meta-analysis. Chin Med J. 2012;125(14):2589-97.

40. Wang CL, Tang XY, Chen WQ, Su YX, Zhang CX, Chen YM. Association of estrogen receptor alpha gene polymorphisms with bone mineral density in Chinese women: a meta-analysis. Osteoporosis international : a journal established as result of cooperation between the European Foundation for Osteoporosis and the National Osteoporosis Foundation of the USA. 2007; 18(3):295-305.

41. Ioannidis JP, Stavrou I, Trikalinos TA, Zois C, Brandi ML, Gennari L, Albagha

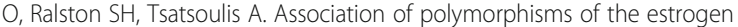
receptor alpha gene with bone mineral density and fracture risk in women: a meta-analysis. Journal of bone and mineral research : the official journal of the American Society for Bone and Mineral Research. 2002;17(11):2048-60.

\section{Submit your next manuscript to BioMed Central and we will help you at every step:}

- We accept pre-submission inquiries

- Our selector tool helps you to find the most relevant journal

- We provide round the clock customer support

- Convenient online submission

- Thorough peer review

- Inclusion in PubMed and all major indexing services

- Maximum visibility for your research

Submit your manuscript at www.biomedcentral.com/submit
) Biomed Central 